\title{
L'ENTREPOSAGE DES BEURRES EN FRIGORIFIQUE \\ INFLUENCE DE LA TENEUR EN CUIVRE SUR L'APTITUDE A LA CONSERVATION (1)
}

\author{
par \\ E. PIRAUX et P. JAMOTTE (2) \\ Station laitière de l'Etat, à Gembloux (Belgique)
}

La plupart des phénomènes d'altération des beurres dans les conditions courantes de conservation étant d'origine biologique et se manifestant sous forme d'hydrolyse de la matière grasse conduisant au rancissement, ou sous forme de dégradation des autres constituants du beurre, on pouvait s'imaginer qu'en entravant par le froid l'action de ces agents d'altération on arriverait à garantir une parfaite conservation, surtout si l'on pousse le refroidissement sensiblement en-dessous du point de congélation.

En réalité, on s'est aperçu que si certains beurres tenus à $-10^{\circ}$ C. se conservaient parfaitement pendant six mois et plus, d'autres, par contre, accusaient au bout de deux ou trois mois des altérations se caractérisant par l'apparition du goût graisseux, du goût suiffeux, du goût huileux, ou du goût de poisson.

Effectivement pourtant, l'action de la plupart des agents d'altération était entravée légèrement au-dessus de $0^{\circ}$, sauf pour certaines moisissures et en cours de conservation à l'état congelé on constatait une destruction progressive de la plus grosse partie de la flore microbienne, surtout de la flore acidophile (ferment lactique - Coli aerogenes - levures), les germes caséolytiques étant encore la catégorie la plus résistante quoique décimée ellemême déjà dans une forte proportion [1".

Cette constatation devait amener rapidement les spécialistes à conjecturer une altération d'un tout autre type, une altération d'ordre chimique, caractérisée essentiellement par des phénomènes d'oxydation des matières grasses ou des phospholipides.

Cette hypothèse cadrait parfaitement d'ailleurs avec les observations que l'on avait pu faire quant à l'action de la flore microbienne qui contrarie l'oxydation du milieu où elle se développe. En supprimant l'action de cette flore, on favorise donc l'intervention des phénomènes d'oxydation.

(1) Conserva, novembre 1953, n० 5 (Moorman's Periodicke, Pers. N.V., La Haye, Hollande).

(2) Les déterminations analytiques dont il est fait mention dans le présent article ont été effectuées en bonne partie par MM. Lheureux et Lacrosse, assistants au Centre de Gembloux, du Comité pour l'Etude scientifidee et technique du Lait et de ses dérivés. 
Bien que certains expérimentateurs [6] aient eru encore en ces derniers temps pouvoir rechercher l'origine du goût de poisson dans des phénomènes d'origine biologique, l'immense majorité des spécialistes s'accordent à voir dans les altérations courantes des beurres en cours de conservation à l'état congelé, uniquement des phénomènes d'oxydation chimique.

L'allure de ces phénomènes d'oxydation est évidemment sous la dépendance de la température. Ces altérations se manifestent d'autant plus tard que la température de conservation est plus basse, et l'on constate d'ailleurs à cet égard une tendance à conserver à température de plus en plus basse : antérieurement, l'entreposage des beurres s'effectuait sur une large échelle à -7 ou $-8^{\circ} \mathrm{C}$. Maintenant on tend à descendre à -11 ou $-12^{\circ} \mathrm{C}$. et on envisage avec faveur des conservations entre -15 et $-20^{\circ} \mathrm{C}$.

L'allure de ces phénomènes d'oxydation et l'intensité de leur action est étroitement sous la dépendance de facteurs bien connus susceptibles d'intervenir comme catalyseurs, et parmi ces facteurs nous citerons la lumière (qui agit surtout par les rayons de la partie invisible du spectre), le $p \mathrm{H}$ du milieu, et certains éléments métalliques pouvant se rencontrer dans les beurres et à ce titre nous citerons surtout le cuivre et le fer. Le manganèse a été redouté également antérieurement, mais à la suite des recherches effectuées ces dernières années $(2,3)$ il ne semble plus que l'on doive lui attribuer un rôle néfaste dans le domaine qui nous occupe. Le fer lui-même est généralement beaucoup moins à redouter que le cuivre [2] : il n'agit qu'à dose beaucoup plus élevée (500 $\gamma$ et plus par kilogramme de beurre) et son action semble se traduire moins par l'apparition des défauts de conservation les plus redoutés (goût de poisson) que par l'apparition d'un goût métallique qui se marquerait parfois déjà dans le beurre frais et pourrait se transformer ultérieurement en goût graisseux [3].

\section{Le cuivre est I'un des principaux catalyseurs de l'oxydation des beurres en cours d'entreposage}

Depuis plusieurs années, les expérimentateurs s'accordent à trouver dans la teneur en cuivre des beurres faits de crèmes fermentées, l'un des facteurs déterminants du comportement en cours de conservation au froid. Nous citerons tout particulièrement à cet égard les recherches des spécialistes hollandais [2, 3]. Ces derniers ont particulièrement fait ressortir la chose par voie statistique et par voie expérimentale.

Nous avons eu l'occasion de réexaminer ce problème à l'occasion des recherches poursuivies au cours des quatre dernières années, sous les auspices du Comité pour l'Etude Scientifique et Technique 
du Lait et de ses Dérivés, avec l'aide de l'I.R.S.I.A., et nous allons synthétiser ici les observations faites au cours de ces recherches dans le domaine particulier qui nous occupe.

En Belgique, la nature de la matière première servant à la préparation du beurre, est très variable suivant les régions. Alors que dans le Nord du pays, les laiteries récoltent du lait qu'elles écrèment dans leurs installations, dans l'Est, une bonne partie des beurreries récoltent des crèmes séparées à la ferme, le ramassage de ces crèmes s'effectuent tous les jours, rarement tous les deux jours, Dans le Sud du pays, par contre, les beurreries ramassent des crèmes qui ont séjourné à la ferme pendant trois ou quatre jours en été, et parfois pendant sept jours en hiver, et qui arrivent done à la laiterie dans un état d'altération assez marqué.

La diversité de la matière première conduit à une forte diversité de qualité de beurre à l'état frais. Ce à quoi nous nous attendions moins, c'est que cette diversité traduise également ses effets dans l'aptitude à la conservation en cours de stockage en frigo, mais dès la première année nous avons été amenés à reconnaître cet état de choses.

Dès lors, nous avons cru sage au cours de l'étude de la conservation en frigo de faire toujours un départ entre ces diverses catégories de beurres, ou, tout au moins, entre les beurres préparés à partir de crèmes assez fraîches, et les beurres préparés à partir de vieilles crèmes.

Les teneurs en cuivre des beurres rencontrés au cours de nos essais présentaient des variations considérables, allant de 30 à 50 jusque 500 à $700 \gamma$ par kilogramme. Cette variation des teneurs en cuivre s'explique aisément. A côté des variations naturelles de la teneur en cuivre des beurres (MULDER, KRUISHEER, DEN HERDER et VAN GiNkeL [3] ont trouvé dans le beurre des variations naturelles de teneurs en cuivre allant de 40 à $110 \gamma$ ) il faut tenir compte surtout de la contamination des crèmes par le cuivre en provenance des appareils dans lesquels ces crèmes passent ou séjournent en cours de préparation du beurre : pasteurisateurs, réfrigérants, bacs de maturation. Dans certaines laiteries ces équipements sont en cuivre étamé et pour peu que l'étamage disparaisse par places, laissant le cuivre à nu, on constate une forte augmentation de la teneur en cuivre des beurres.

Pour juger de l'état d'oxydation des beurres après des périodes de conservation d'une durée respective de deux mois, de quatre mois et de six mois nous avons procédé aux déterminations de l'indice de peroxyde, le seul indice pratique permettant de juger dans le cas des beurres stockés au froid, de l'état d'oxydation.

Comme au cours de deux premières années, l'indice de peroxyde 
avait été déterminé suivant la technique danoise de la méthode de LEA [1] et au cours des deux dernières années suivant la technique de LofTus HiLls [4], nous nous sommes contentés de reprendre dans le tableau ci-joint (tableau II) les résultats des deux dernières années portant uniquement sur les beurres faits de crèmes soumises à une maturation, beurres d'un JH inférieur à 5,0 .

Dans ce tableau, nous avons indiqué les moyennes d'indices de peroxyde des échantillons rangés dans les diverses catégories de teneurs en cuivre soit teneur inférieure à $50 \gamma$, de 50 à $70 \gamma$, de 70 à $100 \gamma$, de 100 à $150 \gamma$, de 150 à $200 \gamma$, et enfin plus de $200 \gamma$ par kilogramme (le cuivre a été déterminé suivant la méthode de Perrin, Lightwoot et MoIr [5]. Ce tableau suffit à souligner la nette influence du cuivre sur la rapidité des phénomènes d'oxydation et sur l'intensité de ces phénomènes.

\section{RÉSULTATS DE CONSERVATION A $-10^{\circ} \mathrm{C}$.}

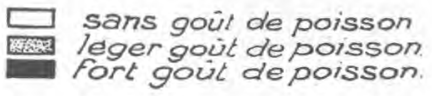

177 beurres de crèmes fraîches, 111 beurres de vieilles crèmes . $1950,1951,1952$ $1950,1951,1952$
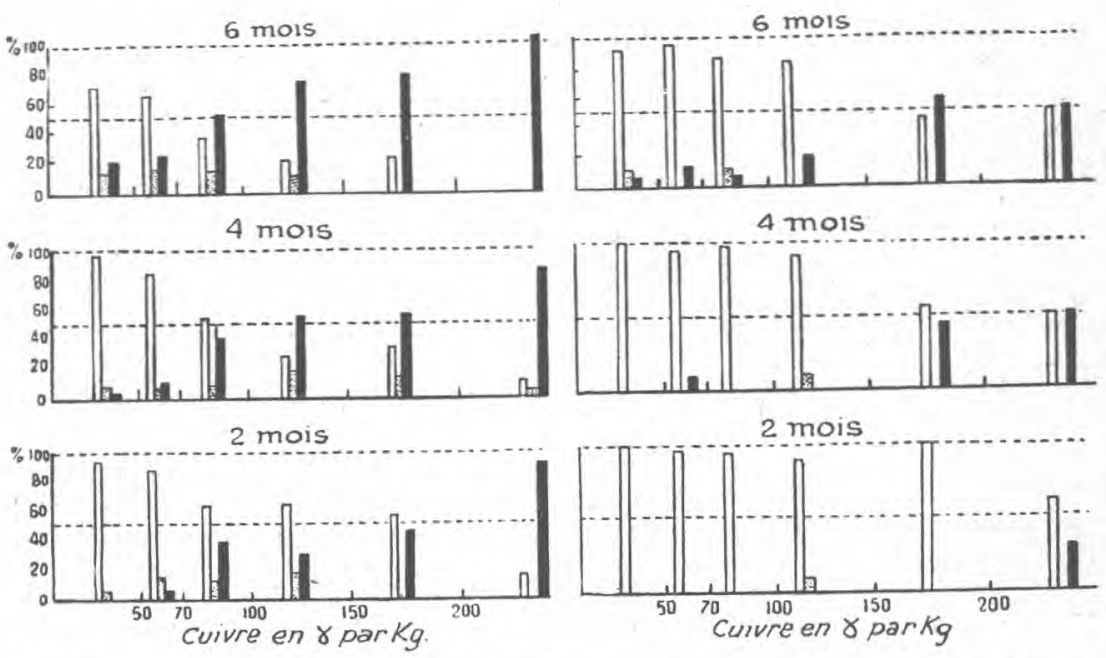

Notons cependant que l'influence de cuivre s'accuse de façon moins sélective dans le cas des beurres faits de vieilles crèmes. Ces beurres présentent en général, même pour les faibles teneurs en cuivre, un état d'oxydation assez caractérisé, mais cette oxydation s'exalte moins au fur et à mesure de l'augmentation de la teneur en cuivre. 
INDICES MOYENS DE PEROXYDE APRÈS 2,4 ET 6 MOIS A $-10^{\circ} \mathrm{C}$.

\section{Beurpes de vieilles oremes}

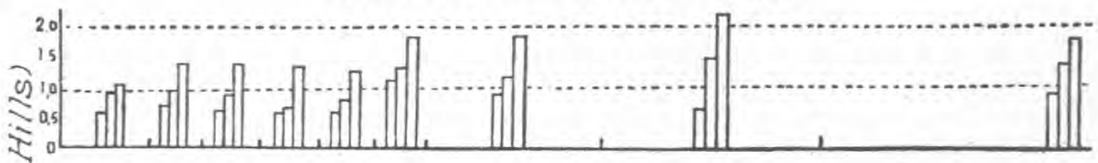

c)

Beurres de erèmes fraiches

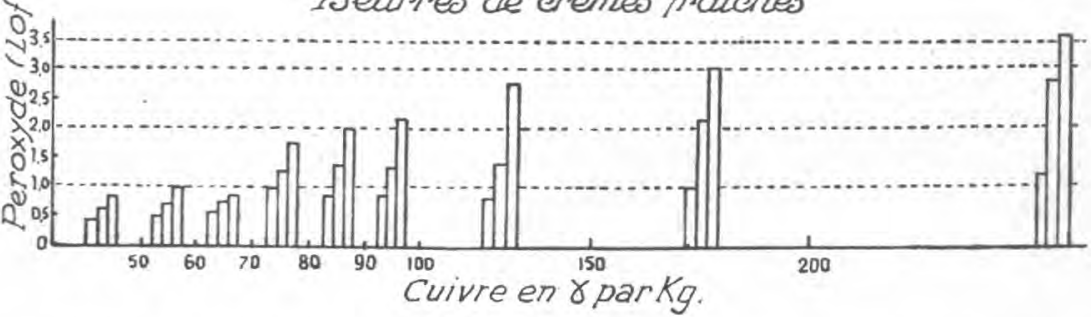

Cette constatation de l'influence du cuivre comme catalyseur d'oxydation dans le cas des crèmes fraîches, nous avons d'ailleurs pu à maintes reprises la vérifier expérimentalement en préparant des beurres à partir des mêmes crèmes d'une part dans dẹs installations de laiteries, installations défectueuses et, d'autre part, dans l'installation expérimentale de la Station, installation en acier inoxydable, cc qui nous permettait de ramener la teneur en cuivre des beurres le plus souvent entre 60 et $80 \gamma$, alors que le beurre à la laiterie contenait généralement plus de $150 \gamma$, parfois plusieurs centaines de $\gamma$ par kilogrammes.

Le tableau ci-après résume le résultat des essais caractéristiques. Il convient d'ailleurs de noter que pour les beurres expérimentaux, dans beaucoup de cas, le goût de poisson était très léger.

\section{Tombre d'essais \\ Goûts de poissons après :}

Nombred'essais 2 mois 4 mois 6 mois

\begin{tabular}{|c|c|c|c|}
\hline Beurres faits à la laiterie .......... & 9 & 7 & 9 \\
\hline $\begin{array}{r}\text { Beurres de la laiterie expérimen- } \\
\text { tale } \ldots \ldots \ldots \ldots \ldots \ldots \ldots \ldots \ldots\end{array}$ & 9 & 1 & 2 \\
\hline
\end{tabular}

Dans les beurres de crèmes fraîches tout particulièrement, cette oxydation conduit à l'apparition du goût de poisson

Nous venons de constater une nette différence entre beurres de crèmes fraîches, et beurres de vieilles crèmes fermières, quant à l'effet du cuivre sur l'intensité des phénomènes d'oxydation dont le beurre est le siège en cours de conservation.

Cette différence va s'accuser tout aussi nettement lorsque l'on envisage la nature des altérations organoleptiques qui apparaissent suite à ces phénomènes. 
Dans les beurres de crèmes fraîches, le goût de poisson s'accuse très rapidement et presque infailliblement dès que l'oxydation atteint un certain stade.

Dans les beurres de vieilles crèmes fermières, on rencontre des altérations de goût beaucoup moins nettes, moins accusées, qui évoluent vers le goût de suif ou le goût graisseux, beaucoup plus rarement vers le goût de poisson, sauf pour certains beurres à teneurs en cuivre vraiment exagérées.

Le graphique ci-après illustre d'ailleurs éloquemment cette situation. Il synthétise les pourcentages de cas d'apparition de goût de poisson après des conservations de deux mois, de quatre mois ou de six mois à - $10^{\circ} \mathrm{C}$., d'une part pour les beurres faits à partir de vieilles erèmes, et cela pour diverses catégories de teneurs en cuivre.

Ces moyennes ont été établies pour des beurres de crèmes fermentées en provenance des diverses régions du pays, et résultent d'essais effectués au cours des années 1950-1951 et 1952.

Dans les beurres de crèmes fraîches, l'influence de la teneur en cuivre sur l'apparition du goût de poisson apparaît de façon très claire. Il n'y a guère que les beurres présentant une teneur en cuivre inférieure à $70 \gamma$ par kilogramme qui présentent des chances sérieuses de bonne conservation durant six mois, tout en présentant encore un pourcentage de déchets assez notable. Entre 70 et $100 \gamma$, on ne peut guère envisager une conservation que de l'ordre de deux à trois mois et entre 100 et $200 \gamma$, de deux mois tout au plus.

Toutes ces données ne sont évidemment que des moyennes et il y a lieu d'étudier chaque cas en particulier.

Dans les beurres faits de vieilles crèmes, la situation apparaît sous un tout autre aspect. Il n'y a guère qu'au-dessus de 150 que l'on constate pour des durées de conservation déjà assez longues (quatre à six mois) l'apparition d'un fort pourcentage de goûts de poisson.

Il est à noter cependant que dans le cas de ces derniers beurres le tableau fait paraître une situation plus belle qu'elle n'est en réalité, cas si le défaut le plus redoutable ne se montre que dans les cas extrêmes, il y a néanmoins des défauts d'oxydation moins caractérisés qui peuvent intervenir et interviennent assez souvent pour déprécier la valeur de ces beurres, défauts qui apparaissent dans de nombreux cas également, consécutifs à des phénomènes d'oxydation.

La teneur en cuivre est un grand facteur d'appréciation de l'aptitude à la conservation, mais ce n'est pas le seul

Ce qui précède suffit à démontrer l'importance qu'il faut atta- 
cher à la teneur en cuivre des beurres, lorsque l'on envisage l'entreposage frigorifique et le soin avec lequel les laiteries doivent éliminer toutes les sources d'augmentation de la teneur en cuivre du beurre par l'appareillage défectueux. D'ailleurs, on a recours de plus en plus à du matériel en acier inoxydable, ce qui permettra d'améliorer la situation de ce côté.

Est-ce à dire qu'au vu de la teneur en cuivre d'un beurre, on puisse infailliblement en déduire son aptitude à la conservation ?

Dans le cas de teneurs très élevées en cuivre, on peut le plus souvent à coup sûr conjecturer l'échec.

Dans le cas de très faibles teneurs en cuivre, on ne peut, par contre, garantir la réussite. De multiples facteurs interviennent pour déterminer l'aptitude à la conservation, tant en ce qui concerne les facteurs de catalyse de l'oxydation, que les facteurs de résistance, et dans l'état actuel, il n'est pas encore possible de poser un diagnostic immédiat basé uniquement sur des constatations de laboratoire.

\title{
BIBLIOGRAPHIE
}

[1] E. Piraux et P. Jamotte. Bull. de l'Inst. Agron. et des Stat. de Rech. de Gembloux, XVIII, 1950, p. 84.

[2] C. I. Kruishegr, P. C. den Herder, W. Werker, J. G. Ginkel et Van Neth. Milk and Dairy Journal, 3, 1949, 25.

[3] H. Mulder, C. I. Kruisheer, P. C. den Herder, J. G. Ginkel et Van IoND, $3,1949,37$.

[4] Loftus Hills et C. J. Thiel. Dairy Res., 14, 1946, 340.

[5] Perrin, Lightfoot, Moir. Journal Dairy Res., 18, 1948, no 1.

[6] T. Storgards, M. Hietaranta. XiI e Congrès International de Laiterie, $2,1949,280$.

\section{LE LAIT ET L'INDUSTRIE LAITIËRE DANS LE MONDE}

\author{
par \\ G. GENIN \\ Ingénieur E.P.C. \\ ÉTATS-UNIS
}

\section{L'emploi de l'acide sorbique pour réduire le développement des moisissures sur le fromage}

D'après des études entreprises par H. J. Deuel et R. B. AlfiNSlater de l'University of Southern California, l'imprégnation par l'acide sorbique des feuilles d'emballage utilisées pour envelopper le fromage retarde le développement des moisissures sur le fromage. Cet acide gras est sans danger, inodore, sans goût, incolore et cons- 